\title{
Water intake from foods and beverages and risk of mortality from CVD: the Japan Collaborative Cohort (JACC) Study
}

\author{
Renzhe Cui ${ }^{1}$, Hiroyasu Iso ${ }^{1, *}$, Ehab S Eshak ${ }^{1,2}$, Koutatsu Maruyama ${ }^{3}$, Akiko Tamakoshi ${ }^{4}$ \\ and the JACC Study Group \\ 'Public Health, Department of Social Medicine, Osaka University Graduate School of Medicine, 2-2 Yamadaoka, \\ Suita-shi, Osaka 565-0871, Japan: ${ }^{2}$ Department of Public Health and Preventive Medicine, Faculty of Medicine, \\ Minia University, Minia, Egypt: ${ }^{3}$ Laboratory of Community Health and Nutrition, Special Course of Food and Health \\ Science, Department of Bioscience, Graduate School of Agriculture, Ehime University, Ehime, Japan: ${ }^{4}$ Public Health, \\ Department of Preventive Medicine, Hokkaido University Graduate School of Medicine, Sapporo, Japan
}

Submitted 23 October 2017: Final revision received 12 April 2018: Accepted 26 April 2018: First published online 15 August 2018

\begin{abstract}
Objective: To examine the association of water intake with risk of mortality from CVD.

Design: Prospective cohort study.

Setting/Subjects: A total of 22939 men and 35362 women aged 40-79 years enrolled in the Japan Collaborative Cohort (JACC) Study with available data regarding water intake from foods and beverages. The underlying causes of death were determined based on the International Classification of Diseases.

Results: During the median 19.1 years of follow-up, 1637 men and 1707 women died from CVD. There was an inverse trend between high water intake and risk of CVD in both sexes. Compared with participants in the lowest quintile of water intake, the multivariable-adjusted hazard ratios (95\% CI) for mortality from total CVD in the highest quintile of water intake were $0.88(0.72,1.07 ; P$ for trend $=0.03)$ in men and $0.79(0.66,0.95 ; P$ for trend $=0.10)$ in women. Those for CHD were $0.81(0.54,1.21 ; P$ for trend $=0.06)$ in men and $0.60(0.39,0.93 ; P$ for trend $=0 \cdot 20)$ in women. Reduced risk of mortality from ischaemic stroke was also observed among women in the highest water intake quintile: $0 \cdot 70(0 \cdot 47,0 \cdot 99 ; P$ for trend $=0 \cdot 19)$. There was no association between water intake and mortality from haemorrhagic stroke in either sex.

Conclusions: Higher intake of fluids from foods and beverages was associated with reduced risk of cardiovascular mortality in both sexes and reduced risk of ischaemic stroke in women in Japan.
\end{abstract}

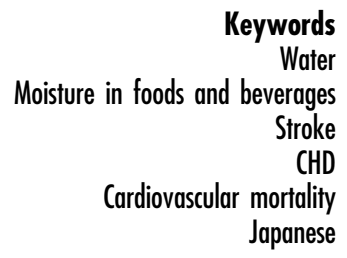

No one can deny how essential water is for life. The human body comprises about $60-75 \%$ water by weight ${ }^{(1)}$. The biological importance of water in man includes its roles in skin moisture ${ }^{(2)}$, thermoregulation ${ }^{(3)}$, cognition enhancement $^{(4)}$, gastrointestinal function and nutrient absorption $^{(5)}$, renal filtration and urinary excretion of blood wastes ${ }^{(6)}$ and cellular homeostasis via maintaining balanced water/mineral content in different body fluids ${ }^{(1)}$.

The human consumption of fluids comes not only from plain water to quench thirst, but also some portion of our daily water intake comes from the moisture in foods and beverages. The proportion of plain water intake to total water intake differs among populations, depending on various factors such as sex, age, weight and climate ${ }^{(7-11)}$. However, the WHO recommends a daily water intake of
2.9 litres for men and 2.2 litres for women under standard conditions $^{(12)}$.

Evidence has been ranked from weak to strong regarding the association of good hydration with reduced risks of different chronic diseases including chronic kidney disease $^{(13)}, \mathrm{CHD}^{(14)}$ and total mortality ${ }^{(15)}$, and it was estimated that a $20 \%$ loss of the body's water content can lead to death ${ }^{(16)}$. A specific importance of water is its impact on the cardiovascular system because water input/ output regulates blood volume, which correlates with blood pressure and heart rate ${ }^{(6,17)}$. Moreover, it was shown in both normotensive and hypertensive individuals that water intake acutely increases the blood pressure while reducing the heart rate ${ }^{(17)}$. Those effects are caused by enhancing the sympathetic nervous system and 
preventing vasovagal reaction rather than changing the blood volume ${ }^{(18,19)}$.

Therefore, the aim of the present study was to examine the association between water intake from foods and beverages and risk of cardiovascular mortality. We gave specific attention to the association with cardiovascular mortality because the water level in the body is associated with vascular contractility via controlling arginine vasopressin hormone ${ }^{(20)}$. Yet, current evidence on the water intake-cardiovascular mortality association is inconclusive. Some studies reported an inverse association between water intake and mortality from $\mathrm{CVD}^{(14,21)}$, while other studies showed no association ${ }^{(13,15,22,23)}$.

We hypothesized that higher water intake from foods and beverages is associated with lower risk of mortality from CVD and tested this hypothesis in a large cohort study of Japanese men and women who participated in the Japan Collaborative Cohort (JACC) Study.

\section{Participants and methods}

\section{Study population}

The JACC Study is a large prospective study started in 1988-1990 and included a total of 110792 participants (46465 men and 64327 women) aged 40-79 years from forty-five Japanese communities. Informed consent was obtained from participants or community leaders, and the ethics committees of Hokkaido University and Osaka University approved the protocol of this study. The sampling methods and protocols of the JACC Study are described in detail elsewhere ${ }^{(24)}$.

A total of 24366 men and 37421 women completed a self-administered questionnaire about their lifestyles and medical histories that included a forty-food-item FFQ at baseline $^{(24)}$. We excluded 1427 men and 2059 women who had a self-reported history of CVD or cancer, as well as history of chronic kidney disease. Therefore, 22939 men and 35362 women were enrolled in the present study.

\section{Dietary assessment}

The FFQ asked about the usual food intake frequency over the past year, without specifying portion size. The five possible frequency responses offered for each food item were: rarely, 1-2 times/month, 1-2 times/week, 3-4 times/week and almost every day ${ }^{(24)}$. The consumption of each food item was calculated by multiplying the frequency score of consumption by $0,0.38,1.5,3.5$ and 7·0/week, respectively. Water intake was estimated from all consumed foods and beverages (beef, pork, ham or sausage, chicken, liver, eggs, milk, yoghurt, cheese, butter, margarine, deep-fried foods or tempura, fresh fish, kamaboko (fish paste), dried fish or salted fish, fried vegetables, spinach or garland chrysanthemum, carrots or pumpkins, tomatoes, cabbage or head lettuce, Chinese cabbage, edible wild plants, fungi, potatoes, algae, pickles, preserved foods using soya sauce, boiled beans, tofu, citrus fruits, fruits excluding citrus varieties, sweets, fresh fruit juice, coffee, tea (green tea, Japanese tea and Chinese tea) and alcoholic drinks). We did not have data on plain water intake. Intakes of water and other nutrients were calculated by multiplying the participant's frequency scores by the water and nutrient contents of each food, which were determined via the Japan Food Composition Tables, fifth edition, followed by summing the content of all the food items. The portion size for each food was estimated by a validation study ${ }^{(24)}$. Water intake was adjusted for total energy intake by means of a sex-specific residual method to reduce the influence of energy intake $^{(25)}$. The FFQ was validated by four seasonal $3 \mathrm{~d}$ dietary records for energy-adjusted values and its reproducibility was tested by applying the same FFQ after one year in a sub-sample of eighty-five participants ${ }^{(26)}$. The Spearman rank-correlation coefficient for the validity of the FFQ-estimated water intake from foods and beverages with reference to the dietary-record water intake from foods and beverages was 0.41 and 0.71 for the two FFQ. The estimated mean water intake was $1317 \mathrm{ml} / \mathrm{d}$ according to the FFQ and $1318 \mathrm{ml} / \mathrm{d}$ according to the four seasonal 3 d dietary records.

\section{Mortality surveillance}

Investigators conducted a systematic review of death certificates as part of the mortality surveillance in each community. Mortality data were forwarded to the public health departments of the respective areas before being centralized at the Ministry of Health and Welfare, and the underlying causes of death were coded in accordance with the International Classification for Diseases, 10th revision (ICD-10). Participants who died after moving out of their original communities were treated as censored cases. The primary end points for the current analysis were deaths from stroke (ICD-10 codes I60-I69), haemorrhagic stroke (ICD-10 codes I60-I61), ischaemic stroke (ICD-10 code I63), CHD ( ICD-10 codes I20-I25) and total CVD (ICD-10 codes I01-I99).

\section{Statistical analysis}

Age-adjusted mean values and proportions of mortality risk factors and participants' characteristics across sexspecific quintiles of water intake $(<1053,1053-1250$, $1251-1442$, $1443-1690$ and $\geq 1691 \mathrm{ml} / \mathrm{d}$ for men; $<1036$, 1036-1220, 1221-1393, 1394-1606 and $\geq 1606 \mathrm{ml} / \mathrm{d}$ for women) were calculated and the differences in those variables across the increasing quintiles of intake were tested by ANCOVA and the $\chi^{2}$ test. Statistical analyses were based on mortality during the follow-up period and the person-years of follow-up was defined as the period from submission of the initial baseline questionnaire to death, departure of a participant from his/her original 
community or termination of follow-up at the end of 2009, whichever came first.

Using the lowest quintile of water intake $(<1053 \mathrm{ml} / \mathrm{d}$ for men; $<1036 \mathrm{ml} / \mathrm{d}$ for women) as the reference category, the sex-specific hazard ratios (HR) and their $95 \%$ CI for mortality outcomes were calculated by Cox proportional hazards modelling after adjustment for age. The multivariable model was further adjusted for sex-specific quintiles of BMI, smoking status (never, ex-smoker, current smokers of 1-19 and $\geq 20$ cigarettes/d), alcohol intake category (never, ex-drinker, current drinkers of 1-22, 23-45, 46-68 and $\geq 69 \mathrm{~g}$ ethanol/d), history of hypertension and diabetes (yes or no), hours of exercise (almost never, 1-2, 3-4 and $\geq 5 \mathrm{~h} /$ week), hours of walking (almost never, $1-2,3-4$ and $\geq 5 \mathrm{~h} / \mathrm{d}$ ), hours of sleep $(<6,6-7$ and $\geq 8 \mathrm{~h} / \mathrm{d}$ continuous), education levels (primary school, junior high school, high school, college or more), perceived mental stress (low, medium, high), dietary sugar and fibre intakes ( $\mathrm{g} / \mathrm{d}$, sex-specific quintiles); as well as intake frequencies of fresh fish, vegetables and fruits (almost never, 1-2 times/month, 1-2 times/week, 3-4 times/week, almost every day), green tea (almost never, 1-4 times/week, 1-2, 3-9 and $\geq 10$ times/d) and coffee (almost never, 1-2 times/month, 1-2 and 3-4 times/week, $1-2$ and $\geq 3$ times/d). We conducted tests for trends across quintiles of water intake by assigning median values for each quintile and testing the significance of this variable. We further analysed the data after exclusion of persons who died within the first 5 years of follow-up to examine a potential effect of as-yet-undiagnosed diseases at baseline. Another sensitivity analysis was conducted by excluding participants with a history of diabetes mellitus at the study baseline. We also tested if the associations between the reported water intake and the risk of mortality from CVD varied by the season when the participants responded to the questionnaire. Probability values for statistical tests were two-tailed and $P<0.05$ was regarded as statistically significant. The SAS statistical software package version 9.4 was used for the analyses.

\section{Results}

During the 19.1-year median follow-up for 22939 men, there were 1637 deaths from total CVD that included 720 deaths from total strokes (234 haemorrhagic strokes and 425 ischaemic strokes) and 390 from CHD. The respective numbers of deaths among 35362 women were 1707, 777 (315 and 384) and 305.

Compared with participants in the lowest quintile of water intake, men in the highest quintile were slightly older and consumed more sugar, while women with the highest quintile were slightly younger, more likely to smoke and to practise sports, but less likely to be diabetic and consumed less sugar (Table 1). Water intake was positively associated with intakes of coffee, green tea, fresh fish, vegetables, fruits and fibre and with education levels for both men and women; while it was inversely associated with alcohol consumption, hours of sleep and history of hypertension in men and women. There was no association between water intake and BMI in either sex.

Tables 2 shows age- and multivariable-adjusted HR (and $95 \% \mathrm{CI}$ ) of mortality from CVD according to quintile of water intake from foods and beverages. Compared with participants in the lowest quintile of water intake, both men and women in the highest quintile of intake had lower risks of mortality from CHD and total CVD. In men, the multivariable-adjusted HR $(95 \% \mathrm{CI})$ were $0.81(0.54$, $1.21 ; P$ for trend $=0.06)$ for CHD and $0.88(0.72,1.07 ; P$ for trend $=0.03$ ) for total CVD. The respective values in women were $0.60(0.39,0.93 ; P$ for trend $=0.20)$ and 0.79 (0.66, 0.95; $P$ for trend $=0.10)$. Reduced risk of mortality from ischaemic stroke was also observed for women in the highest quintile of intake: $0.70(0.47,0.99 ; P$ for trend $=$ $0.19)$; but not in men: $0.89(0.60,1.31 ; P$ for trend $=0.68)$. Excluding early deaths within 5 years from baseline or participants with diabetes at baseline did not change the associations materially (see online supplementary material, Supplemental Tables 1 and 2). There were no differences in the reported association between participants who returned the questionnaire in summer, autumn, winter and spring. There was no association between water intake and mortality from haemorrhagic stroke in either sex.

\section{Discussion}

In the present large prospective cohort study, water intake from foods and beverages was associated with reduced risk of mortality from CHD and total CVD in Japanese men and women; moreover, higher water intake was associated with reduced risk of mortality from ischaemic stroke among women but not men. These associations were independent of age, BMI, intakes of coffee, tea, vegetables, fruits and alcohol, total energy intake and other mortality risk factors.

To the best of our knowledge, the present study is the first to show that higher water intake from foods and beverages is associated with reduced risk of mortality from CHD and ischaemic stroke in the Japanese population. Among 120852 men and women aged 55-69 years of the Netherlands Cohort Study during the 10 years of followup, higher total fluid intake (plain water plus moisture from foods and beverages: $>2 \cdot 0 v \cdot<1 \cdot 0$ litres/d) tended to be associated with reduced risk of mortality from total stroke but not from CHD. The multivariable-adjusted HR (95\% CI) for mortality from total stroke were 0.77 (0.45, $1.30)$ in men and $0.60(0.31,1.15)$ in women, and those for CHD mortality were $1.03(0.73,1.47)$ and $1.04(0.67,1.61)$, respectively $^{(21)}$. The Blue Mountains Eye Study of 3858 men and women aged 49 years or older showed that fluid 
Table 1 Age-adjusted baseline risk characteristics according to water intake from foods and beverages among 22939 men and 35362 women aged 40-79 years, Japan Collaborative Cohort (JACC) Study

\begin{tabular}{|c|c|c|c|c|c|c|}
\hline & \multicolumn{5}{|c|}{ Quintiles of energy-adjusted water intake } & \multirow[b]{2}{*}{$P$ for trenc } \\
\hline & 1 (low) & 2 & 3 & 4 & 5 (high) & \\
\hline \multicolumn{7}{|l|}{ Men } \\
\hline Range of water intake (ml/d) & $<1053$ & $1053-1250$ & $1251-1442$ & $1443-1690$ & $\geq 1691$ & \\
\hline No. of subjects & 4587 & 4588 & 4588 & 4588 & 4588 & \\
\hline Age (years) & 55.5 & $55 \cdot 6$ & $55 \cdot 7$ & $56 \cdot 0$ & $56 \cdot 8$ & $<0.001$ \\
\hline $\mathrm{BMI}\left(\mathrm{kg} / \mathrm{m}^{2}\right)$ & $22 \cdot 8$ & $22 \cdot 7$ & $22 \cdot 6$ & $22 \cdot 7$ & $22 \cdot 8$ & 0.754 \\
\hline Alcohol consumption (g/d) & 43.2 & $34 \cdot 4$ & $32 \cdot 7$ & $29 \cdot 1$ & $27 \cdot 5$ & $<0.001$ \\
\hline Current smoker (\%) & $55 \cdot 2$ & 53.4 & $51 \cdot 3$ & 54.5 & $56 \cdot 7$ & 0.067 \\
\hline History of hypertension (\%) & $21 \cdot 8$ & $20 \cdot 2$ & $19 \cdot 2$ & $17 \cdot 0$ & $16 \cdot 2$ & $<0.001$ \\
\hline Diabetes (\%) & 6.5 & $6 \cdot 6$ & 5.9 & 5.9 & $5 \cdot 8$ & 0.036 \\
\hline Coffee intake, $\geq 1$ time/d (\%) & $32 \cdot 4$ & $52 \cdot 6$ & $60 \cdot 2$ & 67.5 & $66 \cdot 5$ & $<0.001$ \\
\hline Green tea intake, $\geq 1$ time/d (\%) & $50 \cdot 1$ & 79.5 & $92 \cdot 1$ & $96 \cdot 9$ & $98 \cdot 3$ & $<0.001$ \\
\hline Vegetable intake (times/week) & $2 \cdot 1$ & $2 \cdot 6$ & $2 \cdot 7$ & 3.0 & $3 \cdot 2$ & $<0.001$ \\
\hline Fruit intake (times/week) & 1.6 & $2 \cdot 4$ & $2 \cdot 7$ & 3.0 & $3 \cdot 4$ & $<0.001$ \\
\hline Fresh fish intake (times/week) & $3 \cdot 3$ & 3.5 & 3.4 & 3.5 & $3 \cdot 8$ & $<0.001$ \\
\hline Walk, $\geq 3 \mathrm{~h} /$ week (\%) & $6 \cdot 7$ & $6 \cdot 8$ & $6 \cdot 9$ & $7 \cdot 0$ & $7 \cdot 2$ & $<0.001$ \\
\hline Sports, $\geq 5 \mathrm{~h} /$ week (\%) & $6 \cdot 6$ & 5.9 & $7 \cdot 1$ & 7.9 & 7.4 & 0.002 \\
\hline Sleep $(h / d)$ & 7.5 & 7.4 & $7 \cdot 4$ & 7.4 & $7 \cdot 4$ & $<0.001$ \\
\hline Fibre intake $(g / d)$ & $8 \cdot 3$ & $8 \cdot 7$ & 8.9 & $9 \cdot 4$ & $10 \cdot 1$ & $<0.001$ \\
\hline Sugar intake (g/d) & 220 & 218 & 222 & 227 & 239 & $<0.001$ \\
\hline College or higher education (\%) & $14 \cdot 2$ & $17 \cdot 8$ & $20 \cdot 2$ & $20 \cdot 1$ & $19 \cdot 6$ & $<0.001$ \\
\hline \multicolumn{7}{|l|}{ Women } \\
\hline Range of water intake $(\mathrm{ml} / \mathrm{d})$ & $<1036$ & $1036-1220$ & $1221-1393$ & 1394-1606 & $\geq 1607$ & \\
\hline No. of subjects & 7072 & 7073 & 7072 & 7073 & 7072 & \\
\hline Age (years) & $56 \cdot 9$ & $56 \cdot 1$ & $55 \cdot 7$ & $56 \cdot 1$ & $56 \cdot 5$ & 0.001 \\
\hline BMl $\left(\mathrm{kg} / \mathrm{m}^{2}\right)$ & $23 \cdot 1$ & $22 \cdot 8$ & $22 \cdot 9$ & $22 \cdot 9$ & 23.0 & 0.844 \\
\hline Alcohol consumption (g/d) & $14 \cdot 6$ & 9.4 & $8 \cdot 7$ & 8.7 & 8.4 & $<0.001$ \\
\hline Current smoker (\%) & $5 \cdot 0$ & $4 \cdot 1$ & $4 \cdot 1$ & 4.6 & 6.4 & $<0.001$ \\
\hline History of hypertension (\%) & $22 \cdot 0$ & $20 \cdot 4$ & $20 \cdot 4$ & $20 \cdot 5$ & $19 \cdot 0$ & $<0.001$ \\
\hline Diabetes (\%) & 4.0 & 3.2 & $3 \cdot 3$ & 3.2 & 3.2 & 0.031 \\
\hline Coffee intake, $\geq 1$ time/d (\%) & $30 \cdot 2$ & $51 \cdot 3$ & $55 \cdot 0$ & $62 \cdot 0$ & 63.6 & $<0.001$ \\
\hline Green tea intake, $\geq 1$ time/d (\%) & 44.5 & $76 \cdot 6$ & $92 \cdot 3$ & $96 \cdot 0$ & $97 \cdot 0$ & $<0.001$ \\
\hline Vegetable intake (times/week) & $2 \cdot 6$ & $3 \cdot 1$ & $3 \cdot 2$ & 3.4 & 3.7 & $<0.001$ \\
\hline Fruit intake (times/week) & $2 \cdot 4$ & $3 \cdot 1$ & $3 \cdot 3$ & 3.6 & $3 \cdot 9$ & $<0.001$ \\
\hline Fresh fish intake (times/week) & $3 \cdot 3$ & 3.5 & 3.5 & $3 \cdot 6$ & $3 \cdot 8$ & $<0.001$ \\
\hline Walk, $\geq 3 \mathrm{~h} /$ week (\%) & $7 \cdot 0$ & $7 \cdot 1$ & $7 \cdot 3$ & $7 \cdot 3$ & $7 \cdot 4$ & $<0.001$ \\
\hline Sports, $\geq 5 \mathrm{~h} /$ week (\%) & $3 \cdot 8$ & 3.9 & 4.5 & 4.8 & 4.7 & $<0.001$ \\
\hline Sleep $(h / d)$ & $7 \cdot 1$ & $7 \cdot 1$ & $7 \cdot 1$ & $7 \cdot 0$ & $7 \cdot 0$ & $<0.001$ \\
\hline Fibre intake $(\mathrm{g} / \mathrm{d})$ & 8.5 & $8 \cdot 6$ & $8 \cdot 7$ & $9 \cdot 0$ & 9.4 & $<0.001$ \\
\hline Sugar intake $(g / d)$ & 209 & 197 & 198 & 198 & 200 & $<0.001$ \\
\hline College or higher education (\%) & $7 \cdot 6$ & 9.4 & $11 \cdot 1$ & 11.8 & 13.0 & $<0.001$ \\
\hline
\end{tabular}

intake from foods and beverages was not associated with cardiovascular mortality; the multivariable-adjusted HR $(95 \% \mathrm{CI})$ in the highest $v$. lowest quartile of water intake $\left(\geq 3 v .<2\right.$ litres/d) was $0.91(0.70,1.19)^{(22)}$. Similarly, the third National Health and Nutrition Examination Survey (NHANES III) indicated that higher total fluid intake (plain water plus moisture from foods and beverages) was not associated with mortality from CVD; the multivariableadjusted HR (95\% CI) in the highest $v$. lowest quartile $(\geq 3.6 v .<2.1$ litres/d) of total fluid intake was $0.99(0.75$, $1 \cdot 29)^{(15)}$.

Regarding plain water intake, on the other hand, during the 6-year follow-up of 20297 American men and women aged 38-100 years of the Adventist Health Study, higher plain water intake was associated with a reduced risk of mortality from CHD; the multivariable-adjusted HR (95\% CI) for the highest plain water intake $(\geq 5 v . \leq 2$ glasses/d) was $0.39(0.22,0.67 ; P$ for trend $=0.0003)$ in men and 0.52 $(0.27,1.03 ; P$ for trend $=0 \cdot 17)$ in women ${ }^{(14)}$. In addition, during the 10-year follow-up of 120852 men and women aged 55-69 years of the Netherlands Cohort Study, high plain water intake $(>500 \mathrm{ml} / \mathrm{d} v$. zero) tended to be associated with a reduced risk of mortality from total stroke; the multivariable-adjusted HR ( $95 \% \mathrm{CI}$ ) was 0.85 $(0.31,2 \cdot 29)$ in men and $0.49(0.19,1.24)$ in women ${ }^{(21)}$.

Many factors interact to determine the amount of fluid intake; one of which is the season. A previous Japanese study reported that water intake was highest in summer $(2331 \mathrm{~g} / \mathrm{d})$ and lowest in winter $(2134 \mathrm{~g} / \mathrm{d})$, and the intake of water from beverages including plain water increased by $8.4 \mathrm{~g} / \mathrm{d}$ while that from foods decreased by $3 \cdot 1 \mathrm{~g} / \mathrm{d}$ with each $1^{\circ} \mathrm{C}$ increase in mean outdoor air temperature ${ }^{(10)}$. In our study, we could not find any effect modification by the season when the questionnaire was administered on the association of water intake from foods and beverages with cardiovascular mortality. The FFQ in our study inquired about the intake over the last 12 months; therefore, the collected dietary intakes including water from foods and 
Table 2 Hazard ratio (HR) and $95 \% \mathrm{Cl}$ of mortality from CHD, total stroke, stroke type and total CVD, according to quintile of water intake from foods and beverages, among 22939 men and 35362 women aged 40-79 years, Japan Collaborative Cohort (JACC) Study

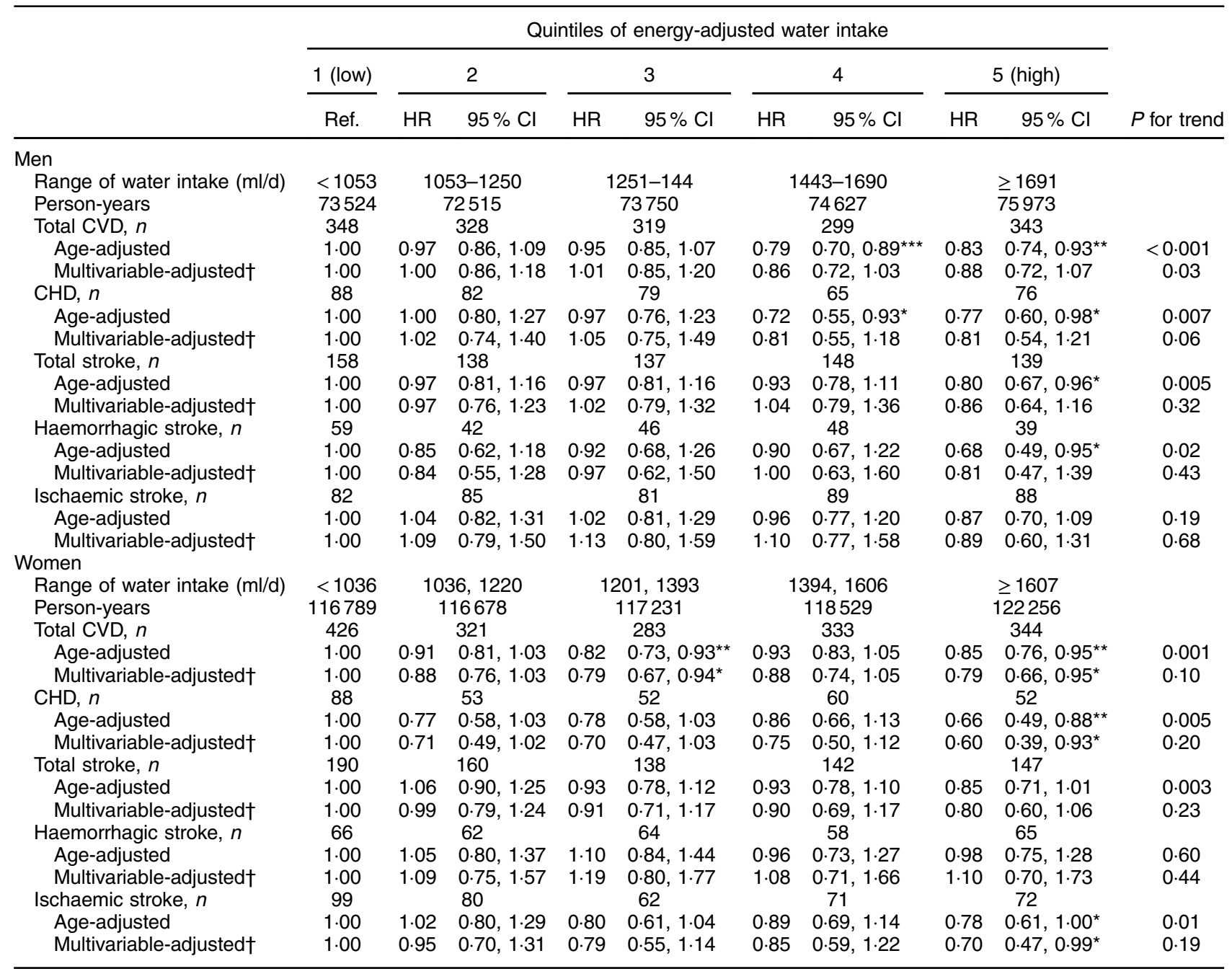

Ref., reference category.

${ }^{\star} P<0.05,{ }^{\star \star} P<0.01,{ }^{\star \star \star} P<0.001$.

†Adjusted for age, BMI (quintile), smoking, alcohol consumption, intakes of coffee, green tea, fresh fish, vegetables, fruits, fibre, sugar and energy, hours of sleep, walking and sports, education level and history of hypertension and diabetes mellitus.

beverages were not confined to a specific season but were the overall intakes over one year.

The proportion of plain water intake out of total fluid intake has been shown to be variable among populations $^{(7-11)}$. For example, this proportion was $33 \%$ in US participants in the NHANES 2005-2006 ${ }^{(7)}, 54 \%$ in Korean participants in the National Health and Nutrition Examination Survey (KNHANES) 2008-2012 ${ }^{(8)}$ and $53 \%$ in adult dwellers of four cities in China ${ }^{(9)}$. Unfortunately, we do not have data on the consumption of plain water in our study to state what percentage is accounted for by plain water. Even the Japan National Health and Nutrition Survey, a survey conducted annually by the Japanese Ministry of Health, Labour, and Welfare to understand the status of people's health, nutritional intake and lifestyle habits, has not published any data about the proportions of plain water and water from foods and beverages. Thus, we were unable to discern any potential differences described in previous studies regarding the associations of water intake from foods and beverages as opposed to plain water intake with cardiovascular mortality. However, small Japanese studies have reported the proportions of water intake from different sources. For example, in a study conducted in four areas in Japan with a total of 121 women aged 30-69 years and 121 men aged 30-76 years who completed $16 \mathrm{~d}$ diet records, the mean total water intake was $2230 \mathrm{~g} / \mathrm{d}$ and almost half of the water was derived from foods $(1130 \mathrm{~g} / \mathrm{d})$, the remaining half coming from beverages including plain water $(1130 \mathrm{~g} / \mathrm{d})^{(10)}$. In another study among 3835 female Japanese dietetic students aged 18-20 years from fifty-three institutions in Japan, the mean total daily water intake $(\mathrm{g} / 4186 \mathrm{~kJ})$ was $1028 \mathrm{~g}$; $654 \mathrm{~g}$ from fluids including plain water and $374 \mathrm{~g}$ from foods ${ }^{(5)}$. A report from thirteen countries that collected $7 \mathrm{~d}$ fluid-specific records showed a total fluid intake from beverages of 1.5 litres/d in 1318 Japanese men and 
women aged 18 years or older, and $18 \%$ of this amount was consumed as plain water ${ }^{(11)}$.

Mechanisms by which water intake is associated with reduced risk of mortality from CVD are not well known. However, the vasopressin system might play a role. Post myocardial infarction activation of the vasopressin system was reported in an observational study of 980 patients. Copeptin, the C-terminal part of the vasopressin prohormone, and N-terminal pro-B-type natriuretic peptide (NTproBNP), an established marker for left ventricular function and the prognosis of myocardial infarction, were at highest levels at admission then plateaued within 3-5d; moreover, their levels were higher in patients who died or were readmitted for heart failure than in survivors ${ }^{(20)}$. Another mechanism was shown in a clinical trial of 293 healthy men and women aged 20-95 years which demonstrated that hypo-hydration leads to high blood viscosity $^{(27)}$, which was positively associated with 8 -year risk of CHD among 933 men aged 45-64 years of the MONICA-Augsburg cohort study ${ }^{(28)}$. Among 4112 men and women aged $\geq 20$ years in NHANES 1999-2006, individuals with high water intakes either from plain water or from foods and beverages had healthier lifestyles, such as high physical activity, high education and high fibre intake, and lower sugar intake, than individuals with low water intakes ${ }^{(29)}$. In the present study, however, the reduced risk of mortality from CVD associated with high fluid intake remained statistically significant after adjustment for education level, fibre and sugar intakes.

The strengths of our study include its prospective design, the use of a validated FFQ, the consistent way of end-point determination and its large sample size from community residents. In addition, the exclusion of persons with known chronic diseases at baseline reduced bias arising from dietary changes due to known morbidity. We even took account of the possibility that preclinical diseases may affect water intake or that prevalent diabetes may increase water intake due to thirst, and we examined the association after excluding early deaths within 5 years from baseline and participants with diabetes at baseline; there was, however, no material change in the findings.

Limitations of the current study include the abovediscussed lack of estimation of total fluid consumption including plain water intake. Unfortunately, none of the previous studies that assessed the associations of intakes of both plain water and water from foods and beverages with risk of $\mathrm{CVD}^{(14,15,21,22)}$ have reported the correlations between plain water intake and that from foods and beverages in their samples. However, among 2691 US adults aged $\geq 20$ years from the NHANES 2005-2006, plain water was positively associated with moisture in foods and inversely associated with moisture in beverages. Moreover, it was suggested that outdoor temperature can affect the proportions of water intake from foods, beverages and plain water ${ }^{(10)}$. Although we found no effect modification by the season when the questionnaire was administered on the association of water intake from foods and beverages with cardiovascular mortality, further studies are needed to investigate the associations between water intakes from various sources and risk of CVD.

\section{Conclusion}

In conclusion, high intake of water from foods and beverages was associated with reduced risk of cardiovascular mortality for both sexes and reduced risk of ischaemic stroke for women in Japan.

\section{Acknowledgements}

Acknowledgements: The authors thank all staff members involved in this study for their valuable help in conducting the baseline survey and follow-up. Financial support: This work was supported by Grants-in-Aid for Scientific Research from the Ministry of Education, Science, Sports and Culture of Japan (Monbusho) and Grants-in-Aid for Scientific Research on Priority Areas of Cancer, as well as Grants-in-Aid for Scientific Research on Priority Areas of Cancer Epidemiology, from the Japanese Ministry of Education, Culture, Sports, Science and Technology (Monbu-Kagaku-sho) (grant numbers 61010076, 62010074, 63010074, 1010068, 2151065, 3151064, 4151063, 5151069, 6279102, 11181101, 17015022, 18014011, 20014026 and 20390156). The funders had no role in the design, analysis or writing of this article. Conflict of interest: None declared. Authorship: H.I. and A.T. designed this research; R.C. and E.S.E. conducted the analyses and prepared the manuscript; H.I., K.M. and A.T. critically revised the manuscript; H.I. had primary responsibility for the final content. All authors read and approved the final manuscript. Ethics of buman subject participation: This study was conducted according to the guidelines laid down in the Declaration of Helsinki and all procedures involving human subjects were approved by the ethics committees of Hokkaido University and Osaka University. Informed verbal consent was obtained from all subjects; in a few study areas, the consent was obtained from the area representative (the 'mayor') after explaining the purpose of the study.

\section{Supplementary material}

To view supplementary material for this article, please visit https://doi.org/10.1017/S1368980018001386

\section{References}

1. Nicolaidis S (1998) Physiology of thirst. In Hydration Throughout Life, p. 247 [MJ Arnaud, editor]. Montrouge: John Libbey Eurotext. 
2. Williams S, Krueger N, Davids M et al. (2007) Effect of fluid intake on skin physiology: distinct differences between drinking mineral water and tap water. Int J Cosmet Sci 29, 131-138.

3. Vogelaere P \& Pereira C (2005) Thermoregulation and aging. Rev Port Cardiol 24, 747-761.

4. Szinnai G, Schachinger H, Arnaud MJ et al. (2005) Effect of water deprivation on cognitive-motor performance in healthy men and women. Am J Physiol Regul Integr Comp Physiol 289, R275-R280.

5. Murakami K, Sasaki S, Okubo H et al. (2007) Association between dietary fiber, water and magnesium intake and functional constipation among young Japanese women. Eur J Clin Nutr 61, 616-622.

6. Schoen EJ (1957) Minimum urine total solute concentration in response to water loading in normal men. J Appl Physiol 10, 267-270

7. Yang M \& Chun OK (2015) Consumptions of plain water, moisture in foods and beverages, and total water in relation to dietary micronutrient intakes and serum nutrient profiles among US adults. Public Health Nutr 18, 1180-1186.

8. Lee KW, Shin D \& Song WO (2016) Total water intake from beverages and foods is associated with energy intake and eating behaviors in Korean adults. Nutrients 8, E617.

9. Ma G, Zhang Q, Liu A et al. (2012) Fluid intake of adults in four Chinese cities. Nutr Rev 70, Suppl. 2, S105-S110.

10. Tani Y, Asakura K, Sasaki S et al. (2015) The influence of season and air temperature on water intake by food groups in a sample of free-living Japanese adults. Eur J Clin Nutr 69, 907-913.

11. Guelinckx I, Ferreira-Pêgo C, Moreno LA et al. (2015) Intake of water and different beverages in adults across 13 countries. Eur J Nutr 54, Suppl. 2, 45-55.

12. World Health Organization (2003) Domestic Water Quantity, Service Level and Health. Geneva: WHO.

13. Sontrop JM, Dixon SN, Garg AX et al. (2013) Association between water intake, chronic kidney disease, and cardiovascular disease: a cross-sectional analysis of NHANES data. Am J Nephrol 37, 434-442.

14. Chan J, Knutsen SF, Blix GG et al. (2002) Water, other fluids, and fatal coronary heart disease: the Adventist Health Study. Am J Epidemiol 155, 827-833.

15. Wu LW, Chen WL, Liaw FY et al. (2016) Association between fluid intake and kidney function, and survival outcomes analysis: a nationwide population-based study. BMJ Open 6, e010708.

16. Popkin BM, D'Anci KE \& Rosenberg IH (2010) Water, hydration, and health. Nutr Rev 68, 439-458.

17. Callegaro CC, Moraes RS, Negrao CE et al. (2007) Acute water ingestion increases arterial blood pressure in hypertensive and normotensive subjects. J Hum Hypertens 21, 564-570.

18. Schroeder C, Bush VE, Norcliffe LJ et al. (2002) Water drinking acutely improves orthostatic tolerance in healthy subjects. Circulation 106, 2806-2811.

19. Lu CC, Diedrich A, Tung CS et al. (2003) Water ingestion as prophylaxis against syncope. Circulation 108, 2660-2665.

20. Khan SQ, Dhillon OS, O'Brien RJ et al. (2007) C-terminal provasopressin (copeptin) as a novel and prognostic marker in acute myocardial infarction: Leicester Acute Myocardial Infarction Peptide (LAMP) study. Circulation 115, 2103-2110.

21. Leurs LJ, Schouten LJ, Goldbohm RA et al. (2010) Total fluid and specific beverage intake and mortality due to IHD and stroke in the Netherlands Cohort Study. Br J Nutr 104, 1212-1221.

22. Palmer SC, Wong G, Iff S et al. (2014) Fluid intake and allcause mortality, cardiovascular mortality and kidney function: a population-based longitudinal cohort study. Nephrol Dial Transplant 29, 1377-1384.

23. Jang S, Cheon C, Jang BH et al. (2016) Relationship between water intake and metabolic/heart diseases: based on Korean National Health and Nutrition Examination Survey. Osong Public Health Res Perspect 7, 289-295.

24. Tamakoshi A, Ozasa K, Fujino Y et al. (2013) Cohort profile of the Japan Collaborative Cohort Study at final follow-up. J Epidemiol 23, 227-232.

25. Willett WC \& Stampfer MJ (1986) Total energy intake: implication for epidemiological analysis. Am J Epidemiol 124, 17-27.

26. Date C, Fukui M, Yamamoto A et al. (2005) Reproducibility and validity of a self-administered food frequency questionnaire used in JACC Study. J Epidemiol 15, Suppl. 1, S9-S23.

27. Beijering RJ, Gips CH, Huizenga JR et al. (1997) Whole blood and plasma water in health and disease: longitudinal and transverse observations and correlations with several different hematological and clinicochemical parameters. Clin Chim Acta 258, 59-68.

28. Koenig W, Sund M, Filipiak B et al. (1998) Plasma viscosity and the risk of coronary heart disease: results from the MONICA-Augsburg Cohort Study, 1984 to 1992. Arterioscler Thromb Vasc Biol 18, 768-772.

29. Kant AK, Graubard BI \& Atchison EA (2009) Intakes of plain water, moisture in foods and beverages, and total water in the adult US population-nutritional, meal pattern, and body weight correlates: National Health and Nutrition Examination Surveys 1999-2006. Am J Clin Nutr 90 , 655-663. 